\title{
WELFARE OF NAIVE AND SOPHISTICATED PLAYERS IN SCHOOL CHOICE
}

\author{
JOSE APESTEGUIA ${ }^{\dagger}$ AND MIGUEL A. BALLESTER ${ }^{\ddagger}$
}

\begin{abstract}
Two main school choice mechanisms have attracted the attention in the literature: Boston and deferred acceptance (DA). The question arises on the ex-ante welfare implications when the game is played by participants that vary in terms of their strategic sophistication. Abdulkadiroglu, Che and Yasuda (2011) have shown that the chances of naive participants getting into a good school are higher under the Boston mechanism than under DA, and some naive participants are actually better off. In this note we show that these results can be extended to show that, under the veil of ignorance, i.e. students not yet knowing their utility values, all naive students may prefer to adopt the Boston mechanism.
\end{abstract}

Keywords: School Choice; Naive Players; Welfare; Veil of Ignorance.

JEL classification numbers: C7; D0; D6.

Date: September, 2011.

$\dagger$ ICREA and Universitat Pompeu Fabra. Department of Economics, Ramon Trias Fargas 25, 08005 Barcelona, Spain. Phone: 003493542 2521. Fax: 003493542 1746. E-mail: jose.apesteguia@upf.edu.

¥ Universitat Autonoma de Barcelona. E-mail: miguelangel.ballester@uab.es. 


\section{INTRODUCTION}

The distinct properties of different school choice mechanisms raise the question of which is the socially optimal. The two that have attracted most attention are the Boston and the deferred acceptance (DA) mechanisms, which differ mainly in that, while in DA it is a dominant strategy for participants to report their ordinal ranking of schools truthfully, under the Boston mechanism students may strategically misreport their rankings at equilibrium. As a consequence, if participants vary in terms of their strategic sophistication, naive players may suffer more under the Boston mechanism, in terms of ex-ante welfare, possibly making the DA mechanism more attractive from an efficiency perspective. ${ }^{1}$

Remarkably, a recent paper, Abdulkadiroglu, Che and Yasuda (2011), shows that although sophisticated players generally do better than naive ones with the same utilities, naive players do not necessarily come off worse under the Boston mechanism. In particular, it shows that the chances of naive participants getting into a good school are higher under the Boston mechanism than under DA, and some naive participants are actually better off under the Boston mechanism. The reason for this result is that the Boston mechanism is such that it allows participants to transmit information on the intensities of their preferences resulting in sophisticated and some naive students gaining an advantage. ${ }^{2}$

In this note, we show, by means of a stylized model, that the results of Abdulkadiroglu, Che and Yasuda (2010) can be extended to show that, under the veil of ignorance, i.e. students not yet knowing their utility values, not only all the sophisticated students, but also all naive ones may prefer to adopt the Boston mechanism.

\section{Model And Results}

There are continuously many students with measure 1 , and three schools denoted by 1,2 and 3. Let $q_{1}, q_{2}>0$ and $1-q_{1}-q_{2}>0$ denote the capacity measures in the three schools. Students have vNM utility values $1, v$ and 0 for schools 1,2 and 3, respectively, with $v \in\{L, H\}, 0<L<H<1$. The measure of the high-type students, the $H$-students, is denoted by $h$. Notice that all students share the same ordinal preferences over schools, and that we assume that the schools have no priorities over students.

We study two mechanisms for the assignment of students to schools, deferred acceptance (DA) and Boston. These can be introduced as follows. In both cases, every student reports an ordinal ranking of schools, and gets a randomly assigned real number in the unit interval such that no two students get the same number. In the first round under DA, following the increasing order of the real numbers assigned, students are tentatively matched to the school on the top of their ranking, until either the capacity of the school is reached or there are no more students ranking first the school. In step $t>1$, students that were not tentatively

\footnotetext{
${ }^{1}$ See Pathak and Sonmez (2008) for a formalization of this argument.

${ }^{2}$ See Apesteguia, Ballester and Ferrer (2011) for a study of the transmission of cardinal utilities in general collective choice problems. See also Miralles (2008) for a discussion on a modification of the Boston mechanism that protects naive students.
} 
matched to any school in step $t-1$ are considered for their $t$-th best school in their ranking, together with all the students that had been tentatively matched to that school in step $t-1$. Then, all these students are tentatively matched to the school by obeying in increasing order the assigned real numbers. The process stops when all students are matched. It is important to remark that this process makes a dominant strategy for students to reveal their true ordinal ranking.

Under the Boston mechanism, following the increasing order of the real numbers assigned, students are definitely matched to the school in the top of their ranking, until either the capacity of the school is reached or there are no more students ranking first the school. In step $t>1$, a student unmatched to any school in step $t-1$ is considered for her $t$-th best school, provided there is excess capacity in the school. Students in this situation are definitely matched by obeying the assigned real numbers in increasing order of magnitude. The process stops when all students are matched. Clearly, this process is manipulable since the truthful revelation of the ordinal preferences is not necessarily a dominant strategy.

In an environment with naive and sophisticated players, DA never harms naive students since truth-telling is always an optimal strategy, but Boston may do. In our setting there is a measure $s$ of sophisticated players that behave strategically. These are the players that may not submit the true ordinal ranking of schools. There is complete information on both the students' types and their degrees of sophistication. Given the mechanisms, sophisticated players reveal their true ranking under DA, but do not necessarily do so under Boston. Sophisticated players under Boston may reveal the true ranking or a ranking with school 2 at the top, followed by 1 , and then by 3 . The remaining measure of students is composed by naive students and always reveal their true ranking of the schools. We analyze symmetric Nash equilibria in pure strategies. We assume that naive and sophisticated students have the same measure of high-type students, that is there is a measure $s h$ of sophisticated-high students and $(1-s) h$ of naive-high students.

Under DA, all students reveal their true preferences and hence, given the random assignment of students to schools, the expected payoff for any student is $q_{1}+v q_{2}$. Under Boston, we are interested in knowing when the sophisticated-high students misreport their ranking, assuming that the parameters of the game guarantee that sophisticated-low students always reveal their true ranking. Proposition 1 shows that there are only two possible cases, depending on the quota of school 2. If school 2 can accommodate all the sophisticated-high students and hence guarantee sure payoffs of $H$ to misreporters, then these students misreport if $H$ is above the expected payoffs for truth-telling when all other sophisticated-high students misreport $\frac{q_{1}}{1-s h}+H\left(1-\frac{q_{1}}{1-s h}\right) \frac{q_{2}-s h}{1-q_{1}-s h}$, and hence if $H>\frac{q_{1}}{1-q_{2}}$ all sophisticated-high students will misreport. However, if school 2 cannot accommodate all the sophisticated-high students, misreporting will result in $H \frac{q_{2}}{s h}$ expected payoffs, provided there are no free slots for them in school 1, while truth-telling when all other sophisticated-high students misreport in this same situation will result in $\frac{q_{1}}{1-s h}$, and hence if $H>\frac{q_{1} s h}{q_{2}(1-s h)}$ all sophisticated-high students will misreport. 
Proposition 1. Let sophisticated-low students reveal their true ordinal rankings. Then, in the Boston mechanism sophisticated-high students misreport their ranking at equilibrium if and only if:

(1) $q_{2} \geq$ sh and $H>\frac{q_{1}}{1-q_{2}}$, or

(2) $q_{2}<$ sh and $H>\frac{q_{1} s h}{q_{2}(1-s h)}$.

Proof of Proposition 1. Consider the Boston mechanism. Assume, first, that $q_{2} \geq s h$. If sophisticated-high students misreport, then there are slots in school 2 for all of them, which gives them an associated payoff of $H$. Expected payoffs for truth-telling when all other sophisticated-high students misreport are $\frac{q_{1}}{1-s h}+H\left(1-\frac{q_{1}}{1-s h}\right) \frac{q_{2}-s h}{1-q_{1}-s h}$. Note that it must be that $q_{1} \leq 1-s h$ since $q_{1}>1-s h$ together with $q_{2} \geq s h$ would imply that $q_{1}+q_{2}>1$, which is absurd. Then, it is easy to see that $H>\frac{q_{1}}{1-s h}+H\left(1-\frac{q_{1}}{1-s h}\right) \frac{q_{2}-s h}{1-q_{1}-s h}$ if and only if $H>\frac{q_{1}}{1-q_{2}}$.

Now assume that $q_{2}<s h$. If sophisticated-high students misreport, there are not enough slots in school 2 for all of them. This results in two possible cases. First, suppose that $q_{1}<1-s h$, i.e., no sophisticated-high student misreporting her true preferences can end up in school 1. The expected payoffs of sophisticated-high students when misreporting are therefore $H \frac{q_{2}}{s h}$. It is easy to see that these payoffs are above the expected payoffs for truth-telling when all other sophisticated-high students misreport, i.e. $\frac{q_{1}}{1-s h}$, if and only if $H>\frac{q_{1} s h}{q_{2}(1-s h)}$ holds. Part (2) in the statement of the Proposition does not explicitly impose $q_{1}<1-s h$, because this condition is implied by the other two, since $1>H>\frac{q_{1} s h}{q_{2}(1-s h)}>\frac{q_{1}}{1-s h}$. Now suppose that $q_{1} \geq 1-s h$, i.e., there are available slots in school 1 for some sophisticated-high students. The expected payoffs of sophisticated-high students misreporting would therefore be $\frac{q_{1}-(1-s h)}{s h}+H \frac{q_{2}}{s h}$. It is easy to see that these payoffs are above the expected payoffs for truthtelling when all other sophisticated-high students misreport, 1 , if and only if $q_{1}+H q_{2} \geq 1$, which is absurd. This concludes the proof.

More importantly, in what is the main result of this note, we now show that under the veil of ignorance, that is, before participants are aware of their type, not only all sophisticated players, but also all naive players may prefer the Boston mechanism to DA.

Proposition 2. There are parameter configurations for which prior to type awareness, both naive and sophisticated participants prefer the Boston mechanism to DA.

Proof of Proposition 2. We start by analyzing the case of naive players. Under condition (1) of Proposition 1 the expected payoffs to a naive player under the Boston mechanism are $\pi_{B}($ naive $)=h \pi_{B}($ naive - high $)+(1-h) \pi_{B}($ naive - low $)=h\left(\frac{q_{1}}{1-s h}+H \frac{q_{2}-s h}{1-s h}\right)+(1-h)\left(\frac{q_{1}}{1-s h}+\right.$ $\left.L \frac{q_{2}-s h}{1-s h}\right)$, while under DA they are $\pi_{D A}($ naive $)=h \pi_{D A}($ naive - high $)+(1-h) \pi_{D A}($ naive low $)=h\left(q_{1}+H q_{2}\right)+(1-h)\left(q_{1}+L q_{2}\right)$. It can be shown that the former is greater than the latter whenever $h H+(1-h) L \leq \frac{q_{1}}{1-q_{2}}$. Notice that under condition (1) of Proposition $1, H$ is above $\frac{q_{1}}{1-q_{2}}$ by assumption, and it is easy to see that $L$ must be below $\frac{q_{1}}{1-q_{2}}$ for sophisticated-low players to prefer to tell the truth. Hence, the condition may hold. 
Now, under condition (2) of Proposition 1 the expected payoffs to a naive player under the Boston mechanism are $\pi_{B}$ (naive $)=h \frac{q_{1}}{1-s h}+(1-h) \frac{q_{1}}{1-s h}$, while under DA they are $\pi_{D A}($ naive $)=h\left(q_{1}+H q_{2}\right)+(1-h)\left(q_{1}+L q_{2}\right)$. Then, $\pi_{B}$ (naive) $>\pi_{D A}($ naive $)$ if and only if $h H+(1-h) L \leq \frac{q_{1} s h}{q_{2}(1-s h)}$. Again, since $H>\frac{q_{1} s h}{q_{2}(1-s h)}$ by assumption and $L<\frac{q_{1} s h}{q_{2}(1-s h)}$ for sophisticated-low players to prefer to tell the truth, this situation is feasible.

Expected payoffs under the Boston mechanism for sophisticated players under condition (1) of Proposition 1 are $\pi_{B}$ (sophisticated $)=h H+(1-h)\left(\frac{q_{1}}{1-s h}+L \frac{q_{2}-s h}{1-s h}\right)$, while under DA $\pi_{D A}($ sophisticated $)=h\left(q_{1}+H q_{2}\right)+(1-h)\left(q_{1}+L q_{2}\right)$. Since $L<\frac{q_{1}}{1-q_{2}}$, it follows immediately that $\pi_{B}$ (sophisticated) $>\pi_{D A}$ (sophisticated). Similarly, under condition (2) of Proposition $1, \pi_{B}($ sophisticated $)=h \frac{q_{2}}{s h} H+(1-h) \frac{q_{1}}{1-s h}$, while $\pi_{D A}($ sophisticated $)=h\left(q_{1}+H q_{2}\right)+(1-$ $h)\left(q_{1}+L q_{2}\right)$, and hence, since $L<\frac{q_{1} s h}{q_{2}(1-s h)}, \pi_{B}($ sophisticated $)>\pi_{D A}($ sophisticated $)$

As a direct corollary to the proof of Proposition 2, we can conclude that sophisticated players are always better off under the Boston mechanism than under DA, while for naive players it depends on their type. Naive-high players prefer DA to Boston, while naive-low players prefer the reverse, Boston to DA. Consequently, if there are sufficiently many naivelow players, naive players may also prefer ex-ante Boston to DA.

\section{REFERENCES}

[1] Abdulkadiroglu, A., Y-K. Che, and Y. Yasuda (2011), "Resolving Conflicting Preferences in School Choice: The "Boston Mechanism" Reconsidered," American Economic Review, 101:399-410.

[2] Apesteguia, J., M.A. Ballester, and R. Ferrer (2011), "On the Justice of Decision Rules," Review of Economic Studies, 78:1-16.

[3] Miralles, A. (2008), "School Choice: the Case for the Boston Mechanism," mimeo, Boston University.

[4] Pathak, P. A., and T. Sonmez (2008), "Leveling the Playing Field: Sincere and Sophisticated Players in the Boston Mechanism," American Economic Review, 98:1636-52. 